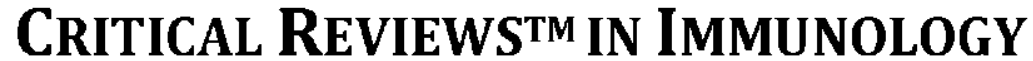

AUTHOR INDEX, VOLUME 38, 2018

Page Range of Issues

Issue 1: 1-78; Issue 2: 79-158; Issue 3: 159-252; Issue 4: 253-342; Issue 5: 343-431; Issue 6: 433-524

Aboulnast, F., 491

Badley, A.D., 491

Bedke, T., 415

Bialoszewska, A., 479

Bom, W.K., 233

Bos, N.A., 145

Boto, P., 63

Bourcue, J., 379

Brockmann, I.., 415

Buart, S., 505

Chen, W., 245

Chirino, L., 105

Chouaib, S., 505

Corneth, O.B.J., 17

Cremer, I., 263

Csuth, T.I., 63

de Roquetaillade, C. 433

Deshmukh, R., 159)

Domingucs, D., 45

Flavell, R.A., 415

Francis, $\Lambda ., 505$

Freen-van Heeren, J.J., 131

lireund-Brown, J., 105

Gagliani, N., 415

Gayed, C., 263

Gossez, M, 433

Hawiger, D., 379

Hendricks, J., 145

Hendriks, R.W., 17

Hervier, B., 263

Ilochmuth, L., 159
Huber, S., 415

Ilutton, A.J., 367

Kambayashi, T., 105

Kaplan, IL.J., 233

Kawai, T., 279

Kimura, T., 403

Kondo, M., 471

Krossi, F.G.M., 145

Kumanogoh, A., 403

Lancioni, C.L.,

Liang, D., 233

Lill, Y., 79

Ma, C., 79

Major, A.S., 333

Malejczyk, J., 479

Metcall, J.P., 303

Miyagawa, I., 471

Mo, P., 245

Momeret, G., 433

Nagoor, N.H., 279

Nakay amada, S., 471

Nawafleh, H.H., 505

Nicolet, B.P., 131

O'Bricn, R.L., 233

Okada, M., 403

Ori, D., 279

Paranjape, G., 491

Paster, B., 343

Patcl. V.I., 303

Pham, I.., 159

Prasad, P., 505
Rezende, R.M., 207

Rhoads, I.P., 333

Rip, J., 17

Shao, II., 233

Sima, C., 343

Sok, S.P.M., 279

Soukou, S., 415

Staples, K.J., 367

Sun, D., 233

Szatmari, I., 63

Tanaka, Y., 471

Teets, A., 159

Terry, S., 505

Tran, E.I.., 159

Uebelhoer, L.S.,

Uzunhan, Y., 263

Van Der Ploeg, F.K., 17

Van Dyke, T.L., 34

Venet, F., 433

Venkatesh, G.H., 505

Vicillard, V., 263

Warner, J.A., 367

Weiner, I1.L., 207

Wolkers, MC., 131

$\mathrm{Yu}, \mathrm{C} ., 245$

Zaarour, R.l., 505

Zeinclabdin, N.A., 505

7hang, P., 453

Zhang, P., 453
Zhang, N, 79

Zhang, N., 79

Zhang, Y., 453
Zhao, B., 253 


\section{CRITICAL ReVIEWSTM IN IMMUNOLOGY}

SubJect IndeX, Volume 38, 2018

Page Range of Issues

Issue 1: 1-78; Issue 2: 79-158; Issue 3: 159-252; Issue 4: 253-342; Issue 5: 343-431; Issue 6: 433-524

\begin{tabular}{|c|c|c|}
\hline adenosine deaminade (ADA), & llow-liSII, 131 & oral tolerance, 207 \\
\hline 233 & Foxp3, 233 & ostcoblasts, 479 \\
\hline adenosine receptors, 233 & growth factor, 471 & osteoclasts, 253 \\
\hline anti-annt-synthetase & gut mucosa, 207 & oxLDL, 333 \\
\hline autoantibody, 263 & host defense, 245 & PD-Ll, 159 \\
\hline antibody, 333 & human mesenchymal stem cells, & phagocytes, 303 \\
\hline antigen presentation, 367 & 471 & plasma dendritic cell, 49l \\
\hline anti-synthetase syndrome, 263 & human, 367 & pulmonary fibrosis, 263 \\
\hline antitumor immunity, 453 & hypoxia, 505 & rat, 145 \\
\hline antitumor response, 505 & $\mathrm{IBD}, 415$ & regulatory $\mathrm{T}$ cell, 379,233 \\
\hline atherosclerosis, 333 & $\mathrm{II}-15,79$ & regulatory $T$ cells, 403,471 \\
\hline autoimmunity, $17,233,263,279$ & IL-33, 453 & respiratory system, 303 \\
\hline ayclumab, 159 & IL-7, 433 & $\mathrm{RN} \Lambda, 13 \mathrm{i}$ \\
\hline B cell receptor, 17 & immunity, 403 & RINX3,63 \\
\hline B cells, 479 & immunoglobulin leavy (IGH) & sepsis, 433 \\
\hline bacteria, 367 & chain genes, 145 & septic shock, 433 \\
\hline bacterial clearance, 245 & immunometabolism, 343 & signal transduction, 105 \\
\hline bacterial infection, 245 & immunoregulation, 379 & smrall-molecule inhibitor, 17 \\
\hline P-cell, 333 & immunotherapy, 105,433 & spleen, 145 \\
\hline bone resorption, 253 & infant, l & $\$ 12,453$ \\
\hline $\begin{array}{l}\text { Bruton's tyrosine kinase, } 17 \\
\text { cancer, } 105,479,505\end{array}$ & $\begin{array}{l}\text { infiection, } 79,105 \\
\text { inflammasome, } 279\end{array}$ & $\begin{array}{l}\text { steroid receptor coactivator-3, } \\
245\end{array}$ \\
\hline $\mathrm{CD} 4,79$ & inflanmmation resolution, 343 & $\mathrm{~T}$ cell differentiation, 379 \\
\hline CD4+ T-cells, 403 & inflammation, 253 & T cell, $1,379,333$ \\
\hline CD8, 79 & inflammatory myopatly, 263 & T' cells, $131,367,479$ \\
\hline CD8+ T cells, 453 & imate and adaptive immune & T-cell activation, $49 \mathrm{l}$ \\
\hline cell differentiation, 63 & cells, 207 & T-cell therapy, 415 \\
\hline cell-mediated cytotoxicity, 479 & innate immunity, 279, 303 & TGl'- $\beta, 79$ \\
\hline cellular signaling, 17 & insulin-like growh factor, 471 & Thl immunity, 1 \\
\hline cGAS-STTNG, 279 & interferon gamma, l & Th17, 233 \\
\hline checkpoint inhibitors, 159 & Lamtorl, 403 & TNF, 253 \\
\hline chondrocytes, 479 & lung, 367 & Toll-like receptor, 279 \\
\hline cytokines, 131 & lymphocyte exhaustion, 433 & TRAlL agonist, 491 \\
\hline cytotoxic T cells, 63 & macrophage, 333 & TR $\wedge$ IL antagonist, 491 \\
\hline cytotoxic T lymphocyte, 491 & macrophages, 403,479 & TRAII, receptor, 491 \\
\hline dendritic cell, 379 & marginal zone $(\mathrm{M} /), 145$ & transcription factors, 63 \\
\hline dendritic cells, $63,453,479$ & memory B cells, 145 & transcriptional proliling, 303 \\
\hline DNA damage, 505 & memory, 79 & Treg cells, 415 \\
\hline DN $\Lambda$ sensors, 279 & Merkel cell carcinoma, 159 & tumor microenvironment, 505 \\
\hline dysbiosis, 343 & monocytes, 303 & tumor necrosis factor, 491 \\
\hline effector function, 131 & natural killer (NK) cells, 105 & type 1 regulatory $\mathrm{T}$ cells, 415 \\
\hline eflector T cell, 379 & natural killer cell, 49l & urothelial cell carcinoma, 159 \\
\hline experimental & neonate, 1 & uveitis, 233 \\
\hline uveitis, 233 & 479 & $\gamma \delta \mathrm{l}$-cell, 233 \\
\hline libroblast, 367 & NTHi, 367 & $\begin{array}{l}\Omega-3 \text { polyunsaturated latty acids, } \\
343\end{array}$ \\
\hline
\end{tabular}




\section{CRITICAL ReVIEWS ${ }^{\mathrm{TM}}$ IN IMMUNOLOGY}

REVIEWERS - 2018

The Editor gratefully acknowledges the cooperation and participation of the reviewers for the year 2018

\begin{tabular}{|c|c|c|}
\hline Bai, X.-Fi. & Jacubzick, C. & Scotton. C. \\
\hline Bcaulicu, $\Lambda$. & Kang, I. & Selva, $\Lambda$ \\
\hline Pergshaken, T. & Kyaw, T. & Simandi, 7. \\
\hline Boer, M. & Li, X. & lakami, M. \\
\hline Bonavida, B. & Menczes, G. & Takcuchi, $\mathrm{O}$. \\
\hline Borir, R. & Moudgil, K. & Taniuchi. I. \\
\hline Caspi, R. & Nitschke, L. & Vanck, $\mathrm{O}$. \\
\hline Charles, J. & Nojima, $\mathrm{S}$. & Wakim, I. \\
\hline Cichocki, ti. & Peronnet, E. & Want, l. \\
\hline Freire, M. & Pillai, S. & Williams, $\Lambda$. \\
\hline Geginat, J. & Puttabyatappa, M. & 7.enewict. $\mathrm{I}_{2}$ \\
\hline Genco, R. & Qin, L. & Zenke, M. \\
\hline Hirata, S. & Rohr, J. & \\
\hline Huang, W. & Satoh, S. & \\
\hline
\end{tabular}


